\title{
Tiburones y rayas capturados por pesquerías artesanales con línea de fondo en el Golfo Dulce, Costa Rica
}

\author{
Andrés López-Garro ${ }^{1} \&$ Ilena Zanella ${ }^{1}$ \\ 1. Asociación Conservacionista Misión Tiburón, Playas del Coco, Guanacaste, Costa Rica; alopez@misiontiburon.org, \\ izanella@misiontiburon.org
}

\author{
Recibido 11-VIII-2014. Corregido 01-XII-2014. Aceptado 14-XII-2014.
}

\begin{abstract}
Sharks and rays caught by artisanal bottom line in Golfo Dulce, Costa Rica. Since May 2010 until May 2011 biological and fishery information regarding 67 artisanal fishing operations were collected in different areas of Golfo Dulce. A total of 30 (44.8\%) onboard operations and $37(55.2 \%)$ landings were observed. During onboard operations, a total of 872 fish were caught: 345 (39.6\%) were sharks (Sphyrnidae, Carcharhinidae, Heterodontidae, Ginglymostomatidae and Triakidae), 228 (26.1\%) "bait" fish (Aridae), 112 (13.1\%) rays (Dasyatidae, Myliobatidae, Rhinobatidae and Mobulidae) and 111 (12.7\%) commercial fish (Lutjanidae, Serranidae Sciaenidae). The capture condition (dead or alive), the low commercial value and the availability of fishermen, allowed us to release 65 sharks and 111 rays, all of them were alive and without hooks. Of these $75.7 \%$ were stingrays (D. longa), $12.6 \%$ eagle ray (A. narinari), 5.4\% cownose rays $(R$. steindachneri), $5.4 \%$ guitar fish ( $R$. leucorhynchus), the spoited ray $2.7 \%$ (U. chilenis), $0.9 \%$ butterfly ray (G. marmorata), and $0.9 \%$ mobula (Mobula sp.). The CPUE rate shows that the sharks and rays contributed more than $50 \%$ of the total catch of fishing operations. However, the months when the fishermen caught more sharks, the rays were not abundant. July and August were the months with the highest shark CPUE, while January-February were the months with the highest ray catches. Regarding the 37 landings observations, a total of 264 sharks were analyzed, being the scalloped hammerhead shark the most abundant ( $S$. lewini, $\mathrm{n}=163$ ), with $61.7 \%$, followed by the common soothhound shark $(M$. lunulatus, $\mathrm{n}=48)(18.2 \%)$, the blacktip shark $(C$. limbatus, $\mathrm{n}=27)(10.2 \%)$ the pacific sharpnose shark $(R$. longurio, $\mathrm{n}=24)(9.1 \%)$, whitenose shark $(0.4 \%)$ (Nasolamia velox, $\mathrm{n}=1)$ and the bonnethead shark $(0.4 \%)$ (Sphyrna tiburo, $\mathrm{n}=1$ ). The fusion of biological and fishery data from onboard observations and landings made it possible to analyze a total 609 sharks belonging to ten species. Of these, $S$. lewini represented more than $51.8 \%$ of total sharks analyzed; $M$. lunulatus, $23.5 \%$ R. longurio, $13.8 \%$ and $C$. limbatus, $8.9 \%$. Other species G. cirratum, G. cuvier, N. velox, shovel head shark (S. tiburo) and the horn shark H. mexicanus were very sporadic since they contributed only $2.0 \%$ of the total. Analyses of length of S. lewini showed that they are mostly juveniles $(74.31 \pm 17.4 \mathrm{~cm})$. Similar situation was found with individuals of $R$. longurio, M. lunulatus and C. limbatus whose mean total lengths were of $65.22 \pm 14.04 \mathrm{~cm}, 94.08 \pm 23.64 \mathrm{~cm} 4.44 \mathrm{~cm}$ respectively 76.65cm. Rev. Biol. Trop. 63 (2): 183-198. Epub 2015 June 01.
\end{abstract}

Key words: Golfo Dulce, Artisanal fisheries, sharks, rays, onboard operations, landings.

El Golfo Dulce se ubica en el Pacífico Tropical Oriental al Sur de Costa Rica, en la provincia de Puntarenas y posee un área de alrededor de $700 \mathrm{~km}^{2}$ (Dean, 1996; Quirós, 2003). Golfo Dulce posee características geomorfológicas muy especiales, que lo convierten en uno de los pocos sistemas de baja circulación descritos para zonas tropicales del mundo (Quesada \& Alfaro, 2004). Con respecto a la diversidad biológica, según Morales (2011) han sido reportadas 1028 especies, distribuidas en 311 familias, donde se incluyen: peces (276 especies), moluscos (296 especies), poliquetos (100 especies) macrocrustáceos (71 especies) y cetáceos (8 especies). Entre estas, figuran especies pelágicas como ballenas jorobadas (Megaptera novaeangliae), tiburones ballena (Rhincodon typus) y delfines (Tursiops 
truncatus, Stenella attenuata), que visitan el golfo periódicamente en busca de refugio y alimentación (Sierra, Vartanian \& Polimeni, 2003; Martínez, Montero \& May-Collado, 2011). Sin embargo, también se reportan diferentes especies que habitan en aguas costeras y que son explotadas con fines turísticos o comerciales por las comunidades vecinas como son los pargos (Lutjanidae), picudos (Isthiphoridae), cabrillas (Serranidae), congrios (Ophidiidae), entre otros (Campos, 1989).

Históricamente, Golfo Dulce ha sido una fuente importante de recursos marino-costeros para comunidades de sus alrededores como La Palma, Golfito, Puerto Jiménez, Zancudo y Pavones (Fargier, Hartmann \& Molina, 2008). Para las pesquerías artesanales de Golfo Dulce, Campos (1989) reportó la captura de una alta diversidad de peces, con más de 175 diferentes especies, mientras que Guzmán y Molina (2007) observaron descargas pesqueras en cuatro comunidades del Golfo y reportaron 68 especies. Sobresalen peces óseos de importancia comercial como las macarelas (Scomberomorus sierra), los robalos (Centropomus pectinatus y Centropomus armatus), los pargos (Lutjanus colorado y Lutjanus guttatus). Además, Campos (1989) y Fargier et al. (2008) reportan también la captura de tiburones de la familia Carcharhinidae y Sphyrnidae, tal es el caso del tiburón punta negra (Carcharhinus limbatus), el tiburón picudo (Rizhoprionodon longurio) y el tiburón martillo (Sphyrna lewini). Otras especies de elasmobranquios que han sido reportadas en las capturas incidentales de Golfo Dulce y que actualmente carecen de valor comercial son las rayas látigo (Dasyatis longa) y la raya gavilana (Aetobatus narinari) (López, 2012). A diferencia de otras comunidades pesqueras del Pacífico de Costa Rica como Puntarenas, Playas del Coco y Quepos, en Golfo Dulce, los tiburones y rayas tienen un bajo valor comercial, por esta razón los pescadores liberan muchas especies de limitada comercialización.

Los elasmobranquios poseen características biológicas muy especiales que los diferencias de los peces óseos como son un crecimiento lento, baja fecundidad y una alta longevidad, lo anterior los vuelve particularmente vulnerables a las pesquerías intensivas y dirigidas tanto a nivel costero como en aguas pelágicas.

Existen diferentes ejemplos de pesquerías donde se reportan disminuciones en las capturas de tiburones y rayas. Tal es el caso de la pesca con palangre o lonlinge en aguas pelágicas del Atlántico canadiense, donde las capturas de tiburón azul (Prionace glauca) en las pesquerías comercial y recreacional han declinado entre el 5-6\% anualmente desde 1995 (Campana, Marks, Joyce \& Kohler, 2006). Así mismo, en el Pacífico Norte, Clarke, Harley, Hoyle y Rice (2013) reportan como las capturas pesqueras entre 1995 y el 2010 declinaron significativamente para el tiburón azul ( $P$. glauca) (5\% por año), para los tiburones makos (Isurus $s p$.) (7\% por año) y para el tiburón punta blanca oceánico ( $C$. longimanus) (17\% por año). Estos ejemplos muestran como en diferentes zonas ha habido una importante disminución en la captura de tiburones y rayas, lo cual puede estar ocurriendo en aguas costarricenses.

A fin de contar con mejor información sobre los recursos marinos existentes en Golfo Dulce, este trabajo caracterizó los elasmobranquios presentes en las capturas de pescadores artesanales que realizan faenas con línea de fondo. Con los resultados obtenidos se recomiendan acciones de manejo precautorias para los principales elasmobranquios (tiburones y rayas) que habitan en Golfo Dulce.

\section{MATERIALES Y MÉTODOS}

Área de estudio: Golfo Dulce se ubica en la provincia de Puntarenas en el Pacífico Sur de Costa Rica entre los $8^{\circ} 27^{\prime}-8^{\circ} 45^{\prime} \mathrm{N}$ y los $83^{\circ} 07^{\prime}-83^{\circ} 30^{\prime} \mathrm{W}$ (Morales, 2011). Por sus características geomorfológicas, ecológicas y considerando la información científica y la brindada por los pescadores sobre sus hábitos pesqueros, se divide en tres áreas de pesca: Golfo Dulce Externo (Zona A), Interno (Zona B) y la zona de Golfito, las cuales están delimitadas como se muestra en el Cuadro 1. 
CUADRO 1

Zonificación del Área Marina de Pesca Responsable Golfo Dulce

TABLE 1

Zonification of the Responsible Fisheries Marine Area of Golfo Dulce

Zonas en el AMPR Golfo Dulce

Golfo Dulce Externo (Zona A): Límite exterior: una línea imaginaria que se extiende desde Punta Banco $\left(08^{\circ}\right.$ 21 ' 56.63" N - 83 $08^{\circ}$ '44.07" W), hasta Cabo Matapalo $\left(08^{\circ} 22^{\prime} 28.73^{\prime \prime} \mathrm{N}-83^{\circ} 17^{\prime} 28.54\right.$ ' W). Límite interior: una línea imaginaria que se extiende desde la margen derecha del río Tigre $\left(08^{\circ} 34^{\prime} 07.52^{\prime \prime} \mathrm{N}\right.$ y $\left.083^{\circ} 19^{\prime} 32.08^{\prime \prime} \mathrm{W}\right)$ hasta Punta Voladera ( $08^{\circ} 37^{\prime} 18.64^{\prime \prime} \mathrm{N}$ y $83^{\circ} 10^{\prime} 55.97^{\prime}$ W).

Golfo Dulce Interno (Zona B): Límite exterior: una línea imaginaria que se extiende desde la margen derecha del río Tigre (08 $\left.34^{\prime} 07.52^{\prime \prime} \mathrm{N}-083^{\circ} 19^{\prime} 32.08^{\prime \prime} \mathrm{W}\right)$ hasta Punta Voladera ( $08^{\circ} 37^{\prime} 18.64{ }^{\prime \prime} \mathrm{N}-83^{\circ} 10^{\prime} 55.97$ ' W). Límite interior: a partir del límite exterior aguas adentro, abarcando todas las aguas del golfo Dulce excepto aquellas áreas sobre las cuales pesa prohibición expresa.

Golfito (Zona Golfito): Límite exterior: una línea imaginaria que se extiende desde Punta Voladera $\left(08^{\circ}\right.$ 37' 18.64" N - 83 10 ' 55.97" W) hasta Puntarenitas de Golfito ( $08^{\circ} 36^{\prime} 36.08^{\prime \prime} \mathrm{N}-083^{\circ} 10^{\prime} 42,12$ ' W). Límite interior: a partir del límite exterior aguas adentro abarcando todas las aguas de la zona de Golfito, excepto aquellas áreas sobre las cuales pesa prohibición expresa. Artes de pesca autorizados zona Golfito:
Artes de pesca permitidos por zona

Pesca de Escama: Cuerda de mano, línea planera (de fondo) y Nasas.

Pesca Deportiva y Turística: Con cañas, carretes y cuerdas de mano.

Pesca de Carnada: Cuerda de mano y cañas y carretes con anzuelo Tipo $\mathrm{J} \mathrm{N}^{\circ} 16$.

Pesca de Ballyhoo (Hemiramphus saltator): Captura con "scoop" o "Dip Net".

Pesca de Escama: Cuerda de mano, anzuelo tipo "J" Número 6 o más y circular igual o mayor $\mathrm{N}^{\circ} 6$, Nasas.

Pesca Deportiva y Turística: Con cañas, carretes y cuerdas de mano y señuelos artificiales de todo tipo. Anzuelos individuales tipo " $\mathrm{J}$ " igual o menor a $\mathrm{N}^{\circ} 6$. Anzuelos individuales circulares igual o mayor a $\mathrm{N}^{\circ} .6$

Pesca de Carnada: Cuerda de mano o cañas y carretes con anzuelo tipo "J" número 16

Pesca de Ballyhoo (Hemiramphus saltator): Captura con "scoop" o "Dip Net".

Pesca de Escama: Cuerda de mano con anzuelo tipo "J" igual o menor a $\mathrm{N}^{\circ} 6$. Anzuelos individuales circulares igual o mayor a $\mathrm{N}^{\circ} 6$.

Pesca Deportiva y Turística: Con cañas, carretes y cuerdas de mano. Se pueden utilizar señuelos artificiales y anzuelos tipo "J" igual o menor a $\mathrm{N}^{\circ} 6$. Anzuelos individuales circulares igual o mayor a $\mathrm{N}^{\circ} 6$.

Pesca de Carnada: Cuerda de mano o cañas y carretes con anzuelo tipo "J" número 16.
Descripción de las pesquerías artesanales en Golfo Dulce: Con base en esta división pesquera y las regulaciones existentes en el AMPR Golfo Dulce, la información analizada en este artículo provienen de faenas realizadas por pescadores artesanales de Puerto Jiménez y Pavones dentro de la Zona A de Golfo Dulce y en sus alrededores. Sobresalen zonas costeras cercanas a diferentes comunidades, playas y ríos conocidas por los pescadores como Río Tigre, Jiménez-Puntarenitas, Zancudo, PilónManzanillo, Punta Banco, Matapalo, entre otros (Fig. 1).

Estas aguas son compartidas por alrededor de 300 pescadores artesanales (Zancudo, Pavones, Puerto Jiménez, Golfito, Pilón, etc.), cuyo objetivo principal son especies comerciales como los pargos (Lutjanidae), cabrillas (Serranidae), congrios (Ophidiidae), etc. Para este fin, se utilizan principalmente la línea de fondo y la cuerda de mano. La línea de fondo consta de una línea madre de nylon de varios kilómetros de largo, de la que cuelgan anzuelos tipo circulares $\left(\mathrm{N}^{\mathrm{o}} 10\right.$ y 12$)$ y J $\left(\mathrm{N}^{\mathrm{o}}\right.$ 7 y 8) aproximadamente cada $5-10 \mathrm{~m}$. Por lo general, las líneas son lanzadas en horas de la noche y madrugada, permaneciendo en el agua por algunas horas hasta su virado. La carnada normalmente consiste en sardinas (Cupleidae), jureles (Carangidae) y peces voladores (Hemiramphidae). Aunque los pescadores dirigen sus esfuerzos hacia especies comerciales, 


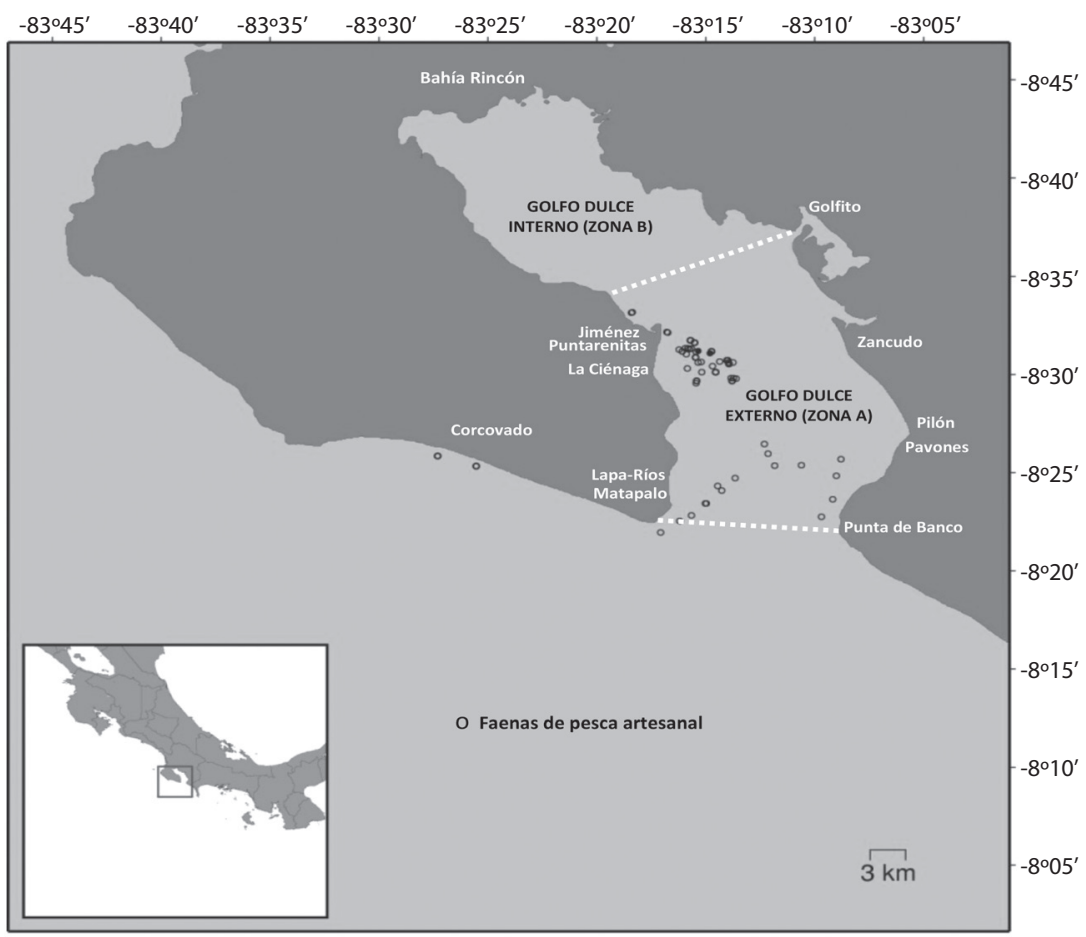

Fig. 1. Localización geográfica de las faenas con línea de fondo realizadas con observador abordo en Golfo Dulce. Costa Rica, 2010-11.

Fig. 1. Geographic location of the fishing operations observed in Golfo Dulce, Costa Rica, 2010-11.

también capturan incidentalmente tiburones (Sphyrnidae, Carcharhinidae y Triakidae) y rayas (Dasyatidae, Myliobatidae, Rhinobatidae y Mobulidae) (López, 2012).

Información Biométrica: La información biométrica procedente de las faenas pesqueras fue recolectada mediante dos fuentes: observación de descargas y observación a bordo. La información biométrica pesquera fue recolectada a lo largo de un año, para lo cual se realizaron dos giras mensuales desde mayo 2010 hasta mayo 2011. Al realizar la observación de descarga, se obtuvieron datos sobre las zonas de pesca según el pescador y sin la utilización de GPS (Sistema de Geoposicionamiento Global) y el número total de tiburones por especie de la captura total descargada. Con los datos de las descargas analizadas se estimaron los aportes porcentuales de las diferentes especies de tiburón descargadas y las zonas donde los pescadores realizaron las faenas.

Al realizar la observación a bordo, se registraron las coordenadas geográficas de la faena de pesca por medio de un GPS, las horas exactas de pesca, el número y tipo de anzuelos calados y un registro del total de individuos por especie de la captura comercial y su condición de captura (vivo o muerto según el estado vital de los individuos en las líneas de pesca) al recoger la línea de pesca. Con las posiciones geográficas de las faenas pesqueras abordo se realizó un mapa; a su vez, se calculó el número de anzuelos y el largo promedio de las líneas de pesca utilizadas por los pescadores. Los individuos analizados fueron agrupados en cinco categorías; tiburones, rayas, carnada (incluye peces óseos utilizados por los pescadores como carnada en faenas posteriores), peces comerciales (incluye especies de peces de interés 
comercial, sobre todo pargos (Lutjanidae)) y anguilas-morenas. Además con los tiburones y rayas recolectados durante las observaciones a bordo se calcularon los porcentajes aportados por cada especie a la captura total y las zonas de pesca utilizadas.

Luego de su identificación taxonómica basado en Fischer et al. (1995), a los tiburones analizados se les tomó la siguiente medida, Longitud Total (LT): medida desde la punta del hocico, hasta el extremo final de la aleta caudal $( \pm 0.1 \mathrm{~cm})$ (Kohler, Casey \& Turne, 1996).

Además, para evaluar diferencias en las proporciones de machos y hembras se utilizó la prueba Chi-cuadrado en el programa Programa InFostat (Di Rienzo et al., 2009) En cuanto a las rayas se registraron las siguientes medidas:
Ancho del Disco (ADi): Medida como la distancia comprendida entre los extremos de las aletas pectorales $( \pm 0.1 \mathrm{~cm})$.

Abundancia Relativa: La abundancia relativa de tiburones y rayas fue estimada a partir de la información recolectada durante las faenas de pesca con línea de fondo y observador a bordo. La Captura por Unidad de Esfuerzo (CPUE) se expresó como el número de tiburones o rayas capturados por anzuelo por hora mensualmente. De tal manera que la CPUE incluyó el tiempo efectivo que los anzuelos estuvieron en el agua, el cual puede variar entre temporadas, entre pescadores e inclusive entre faenas de pesca (Musick \& Bonfil, 2004; López, Arauz, Zanella \& Le Foulge, 2009).

Se utilizó la siguiente ecuación para calcular el número de tiburones o rayas capturados por anzuelo, por hora:

$$
(\text { TTóTR }) \text { CPUE }=(\text { TTóTR } \div \text { TA }) \div(\mathrm{HIV}-\mathrm{HIC}) \div 2+(\mathrm{HFV}-\mathrm{HFC}) \div 2
$$

donde,

$\mathrm{TT}=$ total de tiburones ó $\mathrm{TR}=$ Total de rayas

$\mathrm{TA}=$ total de anzuelos

HIV=hora inicial virado

$\mathrm{HIC}=$ hora inicial calado

HFV hora final virado

$\mathrm{HFC}=$ hora final calado

Los valores de CPUE calculados para los tiburones y rayas capturados en Golfo Dulce, fueron graficados a lo largo del año.

Análisis de datos biométrico-pesqueros de observaciones abordo y de descargas: Los valores de longitud total medio de las especies de tiburones más capturados fueron estimados uniendo los datos provenientes de las observaciones abordo y de las descargas en centros de acopio. Además, se calculó el mínimo (mín), máximo (máx) y la desviación estándar (DE) de las longitudes para las especies de tiburones y rayas más comunes analizados en las observaciones pesqueras.

\section{RESULTADOS}

De mayo 2010 a mayo 2011 se recolectó información biológica-pesquera referente a 67 faenas pesqueras artesanales con línea de fondo, de las cuales 30 (44.8\%) fueron observaciones abordo y 37 (55.2\%) observaciones de descargas en centros de acopio o recibidores. La información referente a observaciones abordo y de descarga fue analizada de manera separada.

Observaciones Abordo: En total se analizaron 30 faenas de pesca con línea de fondo a bordo de embarcaciones artesanales, estas se realizaron en su mayoría $(90 \%)$ en la zona externa de Golfo Dulce y sus alrededores. Sobresalen las zonas conocidas por los pescadores como Los Bajos y Pique Fijo con el $26.7 \%$ y $23.3 \%$ respectivamente, de todas las faenas realizadas. Otras zonas importantes fueron La Ciénaga (20.0\%), Lapa-Ríos (6.7\%), Pavones (6.7\%), Piedra del Sombrero (6.7\%). 
A su vez las zonas de Matapalo Punta Banco y Corcovado fueron menos frecuentadas por los pescadores, ya que solo representaron en conjunto el $9.9 \%$ de las faenas analizadas (Cuadro 2).

En las faenas con observador abordo, los pescadores utilizaron líneas de fondo con una longitud promedio de $2.1 \pm 0.8 \mathrm{~km}$ (mín= $1 \mathrm{~km}$ y máx $=4 \mathrm{~km}$ ). A su vez, en cada faena se lanzaron en promedio un total de $308.1 \pm 90.5$ anzuelos (mín=208 anzuelos y máx=550 anzuelos).

La temperatura del agua superficial marina registrada durante las faenas abordo osciló entre los 25 y $30^{\circ} \mathrm{C}$ a lo largo del año.

Las posiciones geográficas de las 30 faenas de pesca artesanal realizadas con observador abordo en el AMRP Golfo Dulce y sus alrededores se muestran en la Fig. 1. Al mismo tiempo se delimitan la Zona A (Golfo Dulce Externo) y la Zona B (Golfo Dulce Interno), definidas por el Artículo $3^{\circ}$ del Acuerdo de Junta Directiva del INCOPESCA donde se declaró el Golfo Dulce como AMPR (La Gaceta, 2010).

Durante las faenas de pesca con observador abordo se capturaron 872 organismos, de estos, 345 (39.6\%) fueron tiburones Sphyrnidae (Sphyrna lewini, S. mokarran), Carcharhinidae ( $R$. longurio, C. limbatus y Galeocerdo. cuvier), Triakidae (Mustelus lunulatus), 228 (26.1\%) peces clasificados como carnada (Aridae (Arius sp.)), 112 (13.1\%) rayas (Dasyatidae (D. longa), Myliobatidae (Rhinoptera steindachneri), Gymnuridae (Gymnura marmorata) Rhinobatidae (Rhinobatos leucorhynchus), 111 (12.7\%) peces comerciales (Lutjanidae: Lutjanus argentriventis, $L$. novenfaciatus, $L$. gutattus y L.colorado; Serranidae: Epinephelus acanthistius y E. analogus; Sciaenidae: Cynoscion reticulatus). Además se capturaron 63 anguilas y morenas de diferentes especies de las familias Morenidae, respentivamente. Por último durante las observaciones abordo se capturaron "otros" individuos como la tortuga lora (Lephydochelis olivacea), cuya captura fue muy escasa o única (Cuadro 3).

Del total de tiburones capturados $(\mathrm{n}=345)$, el $44.1 \%$ fueron tiburones $S$. lewini, $\mathrm{n}=152$ ),
$27.5 \%$ M. lunulatus $(\mathrm{n}=95), 17.4 \%$ R. longurio $(\mathrm{n}=60), 7.8 \%$ C. limbatus $(\mathrm{n}=27), 2.0 \%$ G.cirratum $(\mathrm{n}=7), 0.6 \%$ G.cuvier $(\mathrm{n}=2), 0.3 \%$ Heterodontus mexicanus (tiburón dormilón, $\mathrm{n}=1)$ y $0.3 \% S$. mokarran ( $\mathrm{n}=1$ ) (Cuadro 4).

La condición de captura de los tiburones durante los viajes de pesca abordo fue variable según la especie y sus hábitos ecológicos. Especies como el tiburón gata ( $G$. cirratum) y el tiburón mamón ( $M$. lunulatus) presentaron una alta sobrevivencia (100.0\% y $98.0 \%$, respectivamente) a la captura. A diferencia especies de mayor natación como el tiburón

\section{CUADRO 2}

Zonas visitadas por los pescadores durante las faenas artesanales con observador abordo. Golfo Dulce, Costa Rica. 2010-11

TABLE 2

Zones used by fishermen during the onboard observations of the artisanal operations. Golfo Dulce, Costa Rica. 2010-11

\begin{tabular}{lcc}
\multicolumn{1}{c}{ Zonas de pesca } & Número faenas & $\%$ del total \\
Los Bajos & 8 & 26.7 \\
Pique Fijo & 7 & 23.3 \\
Jiménez-La Ciénaga & 6 & 20.0 \\
Lapa-Ríos & 2 & 6.7 \\
Piedra del Sombrero & 2 & 6.7 \\
Pavones & 2 & 6.7 \\
Matapalo & 1 & 3.3 \\
Punta Banco & 1 & 3.3 \\
Corcovado & 1 & 3.3 \\
Total & 30 & 100 \\
\hline
\end{tabular}

\section{CUADRO 3}

Total de organismos capturados durante los viajes de observación abordo. Golfo Dulce, Costa Rica, 2010-11

\section{TABLE 3}

Total of individuals caught during the onboard observations of the artisanal operations. Golfo Dulce, Costa Rica. 2010-11

\begin{tabular}{lcc}
\multicolumn{1}{c}{ Grupo } & Número total & $\%$ \\
Tiburones & 345 & 39.6 \\
Carnada & 228 & 26.1 \\
Rayas & 112 & 12.8 \\
Peces comerciales & 111 & 12.7 \\
Anguilas y morenas & 63 & 7.2 \\
Otros & 13 & 1.5 \\
Total & 872 & 100 \\
\hline
\end{tabular}


CUADRO 4

Total de tiburones por especie capturados durante los viajes de observación abordo en Golfo Dulce. Costa Rica, 2010-11

TABLE 4

Total of sharks by species caught during the onboard observations of the artisanal operations. Golfo Dulce,

Costa Rica. 2010-11

\begin{tabular}{llcc}
\multicolumn{1}{c}{ Especie } & \multicolumn{1}{c}{ Nombre común } & Número total & $\%$ \\
Sphyrna lewini & Tiburón martillo común & 152 & 44.1 \\
Mustelus lunulatus & Tiburón mamón común & 95 & 27.5 \\
Rizhoprionodon longurio & Tiburón picudo & 60 & 17.4 \\
Carcharhinus limbatus & Tiburón punta negra & 27 & 7.8 \\
Ginglymostoma cirratum & Tiburón gata & 7 & 2.0 \\
Galeocerdo cuvier & Tiburón tigre & 2 & 0.6 \\
Heterodontus mexicanus & Tiburón dormilón & 1 & 0.3 \\
Sphyrna mokarran & Gran tiburón martillo & 1 & 0.3 \\
Total & & 345 & 100.0 \\
\hline
\end{tabular}

martillo ( $S$. lewini), el tiburón punta negra $(C$. limbatus) y el tiburón picudo ( $R$. longurio) obtuvieron una menor sobrevivencia a la captura $(14 \%, 15 \%$ y $16.5 \%$, respectivamente). Con respecto a la proporción de sexos, por lo general se observaron más machos que hembras; sin embargo, solo para el tiburón martillo dicha diferencia fue significativa (Cuadro 5).

A su vez, durante las faenas con observador abordo se capturaron en total 112 rayas, de las cuales la especie más abundante fue la raya látigo $D$. longa con el $75.7 \%$, seguida por la raya gavilana Aetobatus narinari con $12.6 \%$ y la raya dorada (Rhinoptera steindachneri) con $5.4 \%$. Otras rayas como la guitarra ( $R$. leucorhynchus) y la raya mariposa ( $G$. marmorata) fueron esporádicas y aportaron entre ambas menos del 4\% del total de las capturas (Cuadro 6). Más del $99 \%$ de las rayas capturadas fueron encontradas en las líneas de pesca con vida.
La condición de captura y el bajo valor comercial, permitió liberar con vida y sin anzuelos un total de 65 tiburones. De estos, 30 fueron tiburones martillo (S. lewini), 14 tiburones picudos ( $R$. longurio), 6 tiburones (M. lunulatus), 5 tiburones punta negra (C. limbatus), 7 tiburones gata (G. cirratum), 2 tigres (G.cuvier) y un tiburón dormilón (H. mexicanus). A su vez, la disponibilidad de los pescadores y de la faena pesquera permitió liberar con vida más del $99 \%$ de las rayas capturadas.

Como lo muestran los análisis de captura a bordo de faenas de pesca artesanal, los tiburones y rayas estuvieron presentes prácticamente en todas faenas aportando en conjunto poco más del $50 \%$ del total capturado. Sin embargo, los análisis de las capturas por unidad de esfuerzo (CPUE) muestran cambios en la abundancia de tiburones y rayas a lo largo del año. En los meses donde los pescadores capturaron

\section{CUADRO 5}

Porcentajes de sobrevivencia y proporción sexual para las especies de tiburones más comunes capturadas por las líneas de fondo artesanales. Golfo Dulce, 2010-11

TABLE 5

Survival percentage and sexual proportion for the sharks species more common caught by artisanal bottom lines. Golfo Dulce, Costa Rica, 2010-11

\begin{tabular}{llcccc}
\multicolumn{1}{c}{ Sp } & \multicolumn{1}{c}{ Nombre común } & \% Sobrevivencia & Proporción Sexual & $\mathrm{X}^{2}$ & $p$ \\
Sphyrna lewini & Tiburón martillo común & 14.0 & 1.40 & 0.35 & $<0.05$ \\
Carcharhinus limbatus & Tiburón punta negra & 15.0 & 1.25 & 0.18 & $>0.05$ \\
Rizhoprionodon longurio & Tiburón picudo & 16.5 & 1.10 & 0.13 & $>0.05$ \\
Mustelus lunulatus & Tiburón mamón común & 98.0 & 1.03 & 0.019 & $>0.05$ \\
Ginglymostoma cirratum & Tiburón gata & 100.0 & 1.00 & - & \\
\hline
\end{tabular}


CUADRO 6

Total de rayas por especie capturadas durante los viajes de observación abordo. Golfo Dulce, Costa Rica, 2010-11

TABLE 6

Total of rays by species caught during the onboard observations of the artisanal operations.

Golfo Dulce, Costa Rica. 2010-11

\begin{tabular}{llcc}
\multicolumn{1}{c}{ Sp } & \multicolumn{1}{c}{ Nombre común } & Número total & $\%$ \\
Dasyatis longa & Raya látigo & 84 & 75.7 \\
Aetobatus narinari & Raya gavilana & 14 & 12.6 \\
Rhinoptera steindachneri & Raya nariz de vaca & 6 & 5.4 \\
Urotrygon chilensis & Raya de puntos & 3 & 2.7 \\
Rhinobatos leucorhynchus & Guitarra & 3 & 1.8 \\
Gymnura marmorata & Raya moteada & 1 & 0.9 \\
Mobula sp. & Manta raya & 1 & 0.9 \\
Total & & 112 & 100.0 \\
\hline
\end{tabular}

más tiburones, las rayas fueron poco abundantes y viceversa. Además, en la Fig. 2 se puede observar un definido aumento de la CPUE para ambos grupos. En el caso de los tiburones los bimestres con la mayores CPUE fueron marzoabril y noviembre y diciembre, meses donde se capturaron 0.012 y 0.010 tiburones por anzuelo por hora respectivamente. A su vez, las rayas obtuvieron las mayores CPUE a inicios del año, en los bimestres de enero-febrero y setiembre-octubre cuando se capturaron 0.006 y 0.003 rayas por anzuelo por hora respectivamente (Fig. 2).

Observación de descargas pesqueras: Entre mayo 2010 y mayo del 2011 se analizaron 37 faenas de pesca artesanal con línea de fondo en centros de acopio de Puerto Jiménez y Pavones. De estas, $16.2 \%$ fueron realizadas en la zona conocida Punta Banco, $16.2 \%$ Frente a Jiménez, $13.5 \%$ en Matapalo, $10.8 \%$ en La Ciénaga, $10.8 \%, 10.3 \%$ en Pique Fijo, 10.8\%

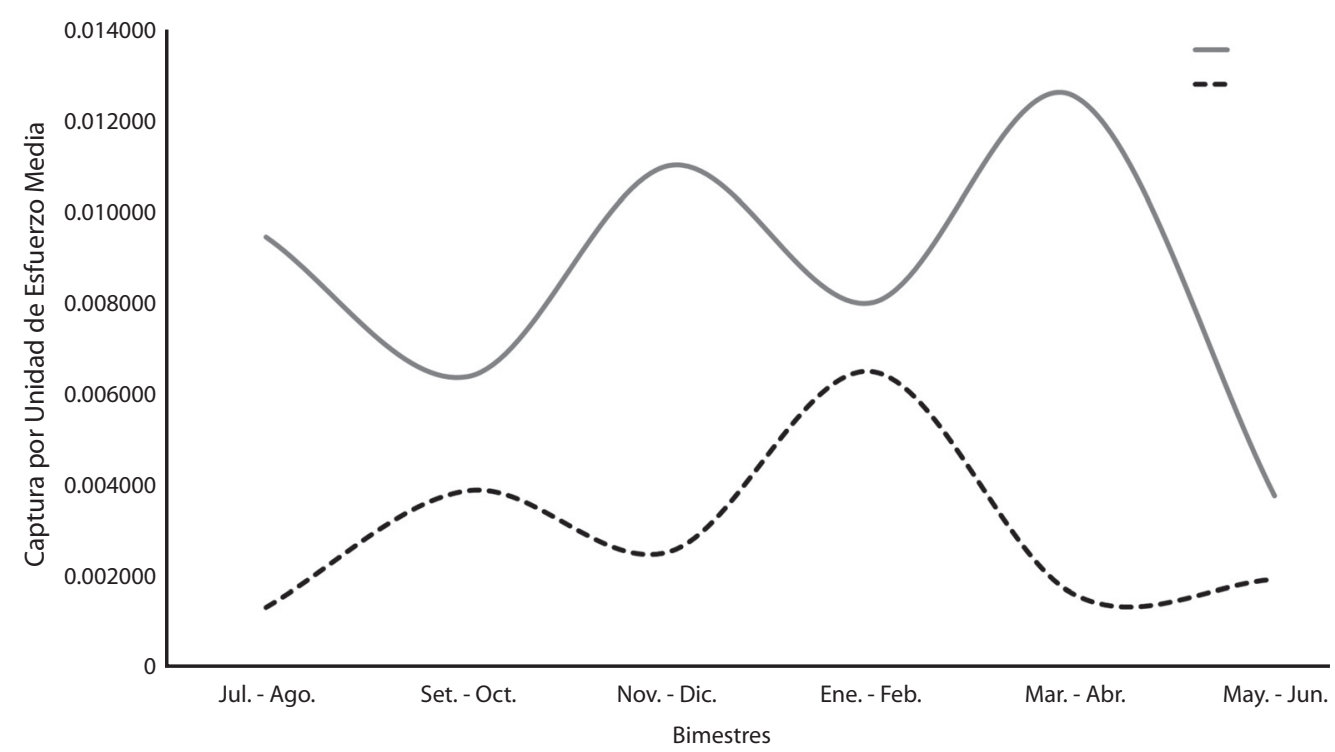

Fig. 2. Abundancia relativa anual expresada como CPUE para los tiburones y rayas en bimestres. Golfo Dulce, Costa Rica, 2010-11.

Fig. 2. Annual Relative abundance, Catch per Unit Effort by bimester, for sharks and rays. Golfo Dulce, Costa Rica, $2010-11$. 
en los Bajos y $10.8 \%$ en Lapa-Ríos. Otras zonas como, Piedra del Sombrero, Pavones y Puntarenitas fueron menos visitadas por los pescadores aportando en total poco más del $10 \%$ (Cuadro 7).

Durante las observaciones de descargas se analizaron en total 264 tiburones, siendo más abundante el tiburón martillo (S. lewini, $\mathrm{n}=163$ ) con el $61.7 \%$, seguido por el tiburón mamón común (M. lunulatus, $\mathrm{n}=48)(18.2 \%)$, el tiburón punta negra $(C$. limbatus, $\mathrm{n}=27)(10.2 \%) \mathrm{el}$ tiburón picudo ( $R$. longurio, $\mathrm{n}=24)(9.1 \%)$, el tiburón fucsia $(0.4 \%)$ (Nasolamia velox, $\mathrm{n}=1)$ y el tiburón cabeza de pala (0.4\%) (Sphyrna tiburo, $\mathrm{n}=1$ ) (Cuadro 8).

\section{CUADRO 7}

Zonas visitadas por los pescadores en las faenas artesanales analizadas en los centros de recibo o acopio de Golfo Dulce. Costa Rica, 2011-11

TABLE 7

Zones used by fishermen during the artisanal operations analyzed in the dock centers. Golfo Dulce. Costa Rica, 2010-11

\begin{tabular}{lcc}
\multicolumn{1}{r}{ Zonas de pesca } & Número faenas & $\%$ del total \\
Punta Banco & 6 & 16.2 \\
Frente a Jimenez & 6 & 16.2 \\
Matapalo & 5 & 13.5 \\
La Ciénaga & 4 & 10.8 \\
Pique Fijo & 4 & 10.8 \\
Los Bajos & 4 & 10.8 \\
Lapa-Ríos & 4 & 10.8 \\
Piedra del Sombrero & 2 & 5.4 \\
Pavones & 1 & 2.7 \\
Puntarenitas & 1 & 2.7 \\
Total & 37 & 100.0 \\
\hline
\end{tabular}

Análisis de datos Biológico-Pesqueros de observaciones abordo y de descargas: $\mathrm{La}$ unión de los datos recolectados en observaciones abordo y en descargas pesqueras, hizo posible analizar un total 609 tiburones pertenecientes a diez especies. De estas, sobresale el tiburón martillo (S. lewini) con más del 51.8\% del total de tiburones analizados, seguido por el M. lunulatus (23.5\%), R. longurio (13.8\%) y $C$. limbatus $(8.9 \%)$. Otras especies como el tiburón G. cirratum, G. cuvier, N.velox, el tiburón cabeza de pala (S. tiburo) y H. mexicanus fueron muy esporádicas ya que aportaron solo el $2.0 \%$ del total.

Los análisis de longitud para las especies de tiburones más abundantes capturadas por los pescadores artesanales de Golfo Dulce revelaron que los tiburones $S$. lewini presentaron longitudes medias de $74.31 \pm 17.41 \mathrm{~cm}$, a su vez los individuos $R$. longurio, M. lunulatus y $C$. limbatus, obtuvieron longitudes totales medias de $65.22 \pm 14.04 \mathrm{~cm}, 94.08 \pm 23.64 \mathrm{~cm}$ y $76.65 \pm 4.44 \mathrm{~cm}$, respectivamente (Cuadro 9).

Para la especie de raya más abundante (D. longa) analizada durante las observaciones de faenas de pesca, se obtuvieron longitudes medias del ancho del disco de $84.63 \pm 12.11 \mathrm{~cm}$ (mín=60cm, máx=119cm).

\section{DISCUSIÓN}

Los análisis de datos pesqueros provenientes de embarcaciones artesanales (2010-2011)

\section{CUADRO 8}

Total de tiburones por especie analizados durante la observación de descargas en puertos de acopio de Golfo Dulce. Costa Rica, 2010-11

TABLE 8

Total of sharks by species analyzed during landings observations in the dock centers. Golfo Dulce. Costa Rica, 2010-11

\begin{tabular}{llcc}
\multicolumn{1}{c}{ Sp } & \multicolumn{1}{c}{ Nombre común } & Número total & \\
Sphyrna lewini & Tiburón martillo común & 163 & 61.7 \\
Mustelus lunulatus & Tiburón mamón común & 48 & 18.2 \\
Carcharhinus limbatus & Tiburón punta negra & 27 & 10.2 \\
Rizhoprionodon longurio & Tiburón picudo & 24 & 1 \\
Sphyrna tiburo & Tiburón cabeza de pala & 1 & 0.1 \\
Nasolamia velox & Tiburón fucsia & 0.4 \\
Total & & 100.0 \\
\hline
\end{tabular}


CUADRO 9

Longitudes medias (LT, cm), mínimo y máximo, para los tiburones más comunes analizados en Golfo Dulce, Costa Rica, 2010-11

TABLE 9

Mean length (LT, cm), minimum and maximum for the more common sharks species analyzed in Golfo Dulce, Costa Rica, 2010-11

\begin{tabular}{clccc} 
N & \multicolumn{1}{c}{ Especie } & LT Media $(\mathrm{cm})$ & LT Min $(\mathrm{cm})$ & LT Max $(\mathrm{cm})$ \\
295 & Sphyrna lewini & $74.31 \pm 17.41$ & 46 & 140 \\
84 & Rizhoprionodon longurio & $65.22 \pm 14.04$ & 43 & 98.5 \\
73 & Mustelus lunulatus & $94.08 \pm 23.64$ & 55 & 140 \\
44 & Carcharhinus limbatus & $76.65 \pm 4.44$ & 72 & 100 \\
\hline
\end{tabular}

que realizan faenas dirigidas a pargos, cabrillas y congrios en distintas zonas del Golfo Dulce y sus alrededores, revelan capturas permanentes de tiburones y rayas tanto en verano como invierno. Esto se refleja claramente en los análisis de faenas abordo, donde casi $40 \%$ de toda la captura fueron tiburones. Altos porcentajes de tiburones han sido reportados en diferentes pesquerías artesanales del mundo. Tal es el caso de Smith, Bizzarro y Cailliet (2009), quienes para las pesquerías artesanales de Baja California (México) reportan que el $50 \%$ de la captura son tiburones. Así mismo, en las proximidades de la Laguna Yahahau, en Quintana Roo, México, Hueter, Castillo, Márquez y Tyminski (2007) reportaron como los tiburones representaron más del $25 \%$ de la captura total de la pesquería artesanal.

En Golfo Dulce sobresalen las capturas de tiburones de las familias Sphyrnidae, Carcharhinidae y Triakidae. Al igual que Golfo Dulce, en el norte de Madagascar un 69\% y $24 \%$ de los tiburones capturados por las pesquerías artesanales pertenecen a las familias Carcharhinidae y Sphyrnidae, respectivamente (Robinson \& Sauer, 2013). De la misma forma en la zona externa del Golfo de Nicoya (Tárcoles) en el Pacifico Central de Costa Rica los tiburones más abundantes en las pesquerías artesanales con línea de fondo para pargo manchado (Lutjanus guttatus) pertenecen a las familias Sphyrnidae, Carcharhinidae, Triakidae (Arauz, López \& Zanella, 2007; López et al., 2009). Estas familias son las comunes en las pesquerías de líneas de fondo por sus hábitos alimenticios demersales o de fondo. Es importante mencionar que a diferencia de Golfo Dulce, donde los tiburones representaron el $40 \%$, en las pesquerías de Tárcoles (Golfo de Nicoya, externo), los tiburones representaron tan solo el 3.5\% de la captura total (López et al., 2009). Esta gran diferencia en los porcentajes de tiburones capturados en las pesquerías de Golfo de Nicoya y Golfo Dulce es muy posible que responda a diferentes amenazas como la contaminación de la cuenca del Río Tárcoles, las construcciones y en especial la sobrepesca del Golfo de Nicoya, ya que estos factores antropogénicos afectan en especial a los tiburones (Camhi, 1999).

De las especies de tiburones presentes en las faenas de pesca artesanal, S. lewini fue la más abundante en la zona externa del Golfo Dulce. Esto coincide con lo reportado por Anislado (2000) que reporta a S. lewini como la especie más abundante en las pesquerías de la costa de Michoacán en México. S. lewini fue la especie de tiburón dominante en las pesquerías con línea en Chennai, India, aportando 37.5\% de todos los elasmobranquios capturados entre 2002 y 2006 (Mohanraj, Rajapakiam, Mohan, Batcha \& Gomathy, 2009). De la misma forma en las pesquerías del norte de Madagascar en Africa, S. lewini representó el $23 \%$ de los tiburones capturados (Robinson \& Sauer, 2013). La utilización de aguas someras como manglares, bahías y bocas de ríos por parte de los juveniles de $S$. lewini y sus habitos alimentios demersales los vuelve muy vulnerables a artes de pesca de fondo. 
Otras especies capturadas en Golfo Dulce fueron el tiburón $M$. lunulatus y $R$. longurio, coincidiendo con lo reportado por Navia, Giraldo y Mejía (2006) en el litoral Pacífico colombiano, donde M. lunulatus representó la segunda especie de tiburón más importante en las capturas. A su vez, Márquez, Corro y Castillo (2005) reportan al género Mustelus $\mathrm{y}$ al tiburón $R$. longurio entre las especies más abundantes en las pesquerías de Sinaloa, México. De igual forma, estas especies son muy comunes y abundantes en otras pesquerías artesanales costarricenses del Pacífico. Tal es el caso de Tárcoles y Golfito donde los tiburones más comunes reportados son $M$. henlei, $M$. lunulatus, $R$. longurio y $N$. velox (Arauz, López, Zanella, Bolaños \& Suarez, 2008; López et al., 2009).

Otro elasmobranquio muy común en las capturas fue la raya $D$. longa, que representó el $75.7 \%$ de todas las rayas capturadas abordo. De la misma forma, en las pesquerías del Golfo de California $D$. longa y D. brevis figuran con importantes capturas en la zona de Sinaloa en invierno (Villavicencio, 1995). A su vez, en aguas costarricense de la parte externa del Golfo de Nicoya, Amador (2010) reportó a $D$. longa como una raya abundante y de importancia en las pesquerías artesanales por su reciente valor comercial.

La presencia y abundancia permanente de tiburones en las pesquerías artesanales de Golfo Dulce revela que las aguas protegidas y productivas del golfo son utilizadas por diferentes especies de elasmobranquios de manera temporal o permanente. Por lo anterior es primordial iniciar estudios a largo plazo para identificar técnicamente, y luego proteger hábitats esenciales a nivel ecológico tales como áreas de crianza, de alumbramiento y de alimentación.

La condición de las especies de tiburones y rayas capturadas en las líneas de fondo fue diferente entre especies, lo anterior relacionado directamente con los hábitos ecológicos de las especies y su fisiología respiratoria (Skomal, 2007). Especies demersales y pocos activas como el tiburón gata ( $G$. cirratum), tienen una alta sobrevivencia a las líneas de pesca ya que poseen barbillas y espiráculos que les ayudan a maximizar el oxígeno durante la respiración. Estos tiburones, al igual que algunas especies rayas, oxigenan sus branquias con un sistema de bombeo en el área bucal (Carlson, Goldman $\&$ Lowe, 2004). De esta forma, cuando son enganchados por un anzuelo logran mantenerse con vida por más tiempo. Por lo anterior más del $99 \%$ de las rayas capturadas fueron encontradas con vida y liberadas.

Por su parte, tiburones con mayor movilidad y que habitan en la columna de agua, como el tiburón picudo ( $R$. longurio), el punta negra (C. limbatus) y el martillo (S. lewini) presentaron una baja sobrevivencia en las líneas de fondo (15\%). Estas especies son más activas y necesitan oxigenar sus branquias manteniendo la boca abierta durante la natación (Carlson et al., 2004), por esto, cuando son capturados logran sobrevivir por poco tiempo ya que se les imposibilita nadar y respirar. Estas diferencias en la sobrevivencia de las especies en Golfo Dulce concuerdan con lo reportado por Morgan y Carson (2010), quienes reportan en el Atlántico de Estados Unidos una mortalidad en las líneas de fondo de $91 \%$ para el tiburón picudo (Rhizoprionodon terraenovae) y $85 \%$ para el tiburón punta negra (Carcharhinus limbatus).

Al contrario, cuando los pescadores artesanales utilizan la cuerda de mano la sobrevivencia de los tiburones y rayas es muy alta (cercana al 100\%), sin importar la especie. Por lo anterior la cuerda de mano es un arte de pesca que permite la implementación de acciones de manejo más efectivas tales como la liberación de juveniles y especies no comerciales, entre otras.

La condición de captura, y la limitada comercialización (bajo valor económico) de la mayoría de especies de tiburones (S. lewini, $G$. cirratum y $R$. longurio), permitió la liberación de la mayoría de individuos con vida. Sin embargo, la situación es diferente para especies como el tiburón mamón ( $M$. lunulatus) ya que aunque tiene una alta sobrevivencia de captura (98\%) en las líneas de pesca de fondo, el alto valor de su carne en el mercado local hace 
que los pescadores no lo liberen (López \& Zanella, 2011).

En cuanto a la rayas, en la actualidad no existe comercialización, por lo que más del 99\% se liberaron con vida. Lo anterior debido sobre todo al difícil acceso que existe entre las comunidades costeras de Golfo Dulce y las grandes ciudades y mercados, al no haber demanda, los pescadores prefieren liberar las especies no comerciales, como son la mayoría de los tiburones y todas las rayas. En otras comunidades del Pacífico de Costa Rica como en Tárcoles, Puntarenas y Cuajiniquil, las rayas son comercializadas para exportar su carne a países de la región como México. Amador (2010) reporta en la comunidad de Tárcoles la utilización de una línea de fondo especializada en la captura de rayas látigo por pescadores artesanales, inclusive esta investigadora reporta como la pesca dirigida hacia esta especie, en solo tres años produjo un descenso de las capturas cercano al $60 \%$, lo que a su vez pudo afectar al stock de manera irreversible.

Al analizar las longitudes totales de los tiburones y rayas más comunes, la mayoría fueron capturados en estados juveniles. Tal es el caso de los tiburones martillo, cuya longitud total media $(74.31 \pm 17.41 \mathrm{~cm})$ fue por debajo de la talla de primera madurez sexual reportada para S. lewini por Branstetter (1987) y Anislado (2000) en aguas del Pacífico Mexicano. El primer autor determinó que en el Golfo de México las hembras maduran a los $250 \mathrm{~cm}$ con 15 años de edad y los machos a los $180 \mathrm{~cm}$ con 10 años de edad. Mientras que, según Anislado (2000) en el litoral de Michoacán de México las hembras maduran a los $190 \mathrm{~cm}$ con 4.4 años de edad y los machos a los $170 \mathrm{~cm}$ con 3.6 años. Lo mismo sucede con $R$. longurio, cuya longitud media de captura fue de $65 \mathrm{~cm}$ y su talla de primera madurez sexual se reporta entre $85-87 \mathrm{~cm}$ para las hembras y $93 \mathrm{~cm}$ para los machos (Márquez et al., 2005).

En cuanto a los tiburones $C$. limbatus analizados, la longitud total media fue cercana a los $75 \mathrm{~cm}$, por lo que todos los individuos fueron juveniles. Según Gaitán y López (2008), esta especie alcanza la madurez sexual entre los 120 y $190 \mathrm{~cm}$ en las hembras, y entre $135 \mathrm{y}$ $180 \mathrm{~cm}$ en los machos.

La situación de la raya $D$. longa fue similar, ya que según López (2009) en el Pacífico de Colombia esta especie alcanza la madurez sexual a los $120 \mathrm{~cm}$ y $92 \mathrm{~cm}$ de ancho de disco para hembras y machos respectivamente, y está siendo capturada en Golfo Dulce con un ancho de disco promedio menor a $85 \mathrm{~cm}$. Sin embargo, Villavicencio, Downton y Meléndez (1994) determinaron la talla mínima de madurez de los machos de $D$. longa de $80 \mathrm{~cm}$ de ancho de disco. Por lo anterior, un alto porcentaje de los individuos de $D$. longa capturados en Golfo Dulce, corresponden a rayas juveniles. El limitado comercio de esta especie en la zona y su alta sobrevivencia en las líneas hizo posible que estas rayas juveniles fueron liberadas con vida en la mayoría de los casos.

Las gráficas de las CPUE de los elasmobranquios capturados en Golfo Dulce, revelan mayores abundancias mensuales de tiburones que de rayas en las pesquerías artesanales. La CPUE de rayas y tiburones muestran una tendencia opuesta, de tal manera que en los meses con mayores abundancias de rayas (enerofebrero y setiembre-octubre) las CPUE de tiburones son bajas. Lo anterior posiblemente debido a cambios ambientales en las aguas y en la dinámica de nutrientes de Golfo Dulce. Sin embargo, debido al bajo número de CPUE por bimestre no se puedo realizar una prueba estadística, por tanto es necesario seguir monitoreando la pesca artesanal en Golfo Dulce y así obtener datos y conclusiones más robustas.

A pesar de esto, la tendencia descrita ha sido reportado para otras zonas como en el Pacífico Central de México, donde Pérez-Jiménez, Sosa-Nishizak y Estrada (2005) reportan las mayores capturas de rayas $(D$. long $a, D$. brevis y $R$. glaucostigma), cuando los tiburones son poco abundantes. De la misma manera los reportes de avistamientos subacuáticos en sitios de buceo del Golfo de Papagayo Costa Rica, reportan las mayores abundancias de $D$. longa durante enero, febrero y marzo (Arauz, López, Zanella \& Bolaños, 2009). En estos meses las aguas del Pacífico Norte costarricense están 
expuestas a fuertes vientos y oleajes que vuelven las aguas más frías y productivas atrayendo diferentes especies marinas, entre estas a $D$. longa. Sin embargo, en estos meses los avistamientos subacuáticos del tiburón punta blanca (Triaenodon obesus) disminuyen (Arauz et al., 2009). Este comportamiento en la CPUE es muy similar al reportado por Arauz et al. (2007) en Tárcoles, donde las mayores capturas de elasmobranquios (tiburones y rayas) se reportan en enero-febrero y setiembre-octubre, influenciadas principalmente por las altas abundancias de tiburones mamones (Mustelus $s p$.) en la época más lluviosa del año. Además, se debe de contemplar que la abundancia relativa o CPUE posee variables específicas que podrían cambiar entre pescadores, como la especie objetivo, condiciones ambientales, eficiencia de la embarcación y la dinámica de la población objetivo (Maunder et al., 2006). Por lo anterior, es necesario monitorear las capturas y el esfuerzo pesquero de los pescadores artesanales en Golfo Dulce a fin de contar con información más robusta que nos permita promover restricciones para ciertas zonas, especies y épocas del año.

\section{CONCLUSIONES Y RECOMENDACIONES PARA LA CONSERVACIÓN Y MANEJO DE LOS TIBURONES Y RAYAS MÁS COMUNES EN GOLFO DULCE}

- Más del $85 \%$ de los tiburones martillo ( $S$. lewini) capturados en la línea de fondo no sobrevive, por esta razón se recomienda regular este arte de pesca en Golfo Dulce, sobre todo en zonas con altas capturas utilizadas por los pescadores como por ejemplo la Ciénaga y Los Bajos (cercanías a Puerto Jiménez). Al igual que S. lewini otras especies de tiburones presentan altas mortalidades en las líneas de fondo (más del 85\%) como es el caso de C. limbatus y $R$. longurio. Para promover la conservación de estas especies se recomienda utilizar líneas de fondo cortas (de 1-2km de largo y con menos de 200 anzuelos) a fin de revisar la línea durante la faena pesquera y liberar estas especies que no tienen valor comercial.

- Se recomienda que en los sitios donde se capturaron un mayor número de tiburones y rayas, como es el caso de la Ciénaga y Los Bajos (cercanías a Puerto Jiménez), se utilice solo la cuerda de mano, ya que con este arte de pesca se ha observado que sobrevive el $100 \%$ de los tiburones y rayas capturadas.

- Se recomienda monitorear las capturas artesanales pesqueras, a fin de implementar vedas temporales durante los periodos donde se reportaron las mayores capturas de tiburones y rayas. Esta recomendación debería basarse en análisis de CPUE mensuales y anuales.

- Con el fin de prevenir la futura comercialización de algunas especies vulnerables de tiburones y rayas, se recomienda prohibir la venta y comercialización en AMPR Golfo Dulce, del tiburón gata $(G$. cirratum), el tiburón punta blanca (Triaenodon obesus) el tiburón tigre (G. cuvier), el tiburón dormilón (H. mexicanus), del tiburón espinoso negro (Echinorhinus cookei), todas especies que actualmente no se comercializan, así como de todas las especies de rayas capturadas incidentalmente (D. longa, A. narinari, $R$. steindachneri, U. chilensis, R. leucorhynchus, G.marmorata, Mobula sp., entre otras.) que a futuro podrían volverse atractivas para el comercio.

- Debido a la poca cantidad de pescadores que faenan en Golfo Dulce, se podrían promover alternativas económicas para los pescadores artesanales, tales como tours de pesca artesanal, avistamiento de ballenas y delfines, tours de manglar, etc. Esto a fin de prohibir (temporal o permanentemente) el uso de la línea de fondo en sitios con altas capturas (ejemplo: frente Punta Banco).

- Se recomienda el uso de anzuelos circulares, que facilitan la liberación de especies no objetivo como los tiburones así como no utilizar reinales ni ningún tipo 
de extensión de metal entre los anzuelos y la cuerda de nylon que fomenten la captura de los tiburones y otras especies no objetivo.

- Es importante tratar de disminuir el esfuerzo pesquero, por tanto se promueven la utilización de líneas cortas que facilitan la revisión y la liberación de ciertas especies altamente vulnerables y no objetivo como lo son rayas y tiburones.

- Se sugiere realizar programas de educación ambiental con pescadores (artesanales, palangreros y deportivos) y con otros miembros de las comunidades con el fin de crear conciencia social y promover actitudes en pro de la conservación de los tiburones y rayas, como por ejemplo la liberación de los individuos capturados vivos durante las faenas de pesca y la protección de las zonas costeras más vulnerables.

- Se recomienda monitorear y dar seguimiento a las pesquerías artesanales de Golfo Dulce, a fin de obtener información básica sobre las captura de elasmobranquios. Para ello debería implementarse un programa conjunto de recolección de datos biológicos básicos entre las instituciones gubernamentales y pescadores artesanales.

\section{AGRADECIMIENTOS}

Este trabajo de investigación fue posible gracias al apoyo de diferentes pescadores artesanales de Golfo Dulce, en especial de Puerto Jiménez y Pavones. José Collado, Pedro Rodríguez, Pedrito Rodríguez, Willian Mata, muchas gracias por su cooperación y por permitirnos analizar tanto abordo como en muelles las capturas de tiburones y rayas de sus faenas artesanales. Además debemos agradecer a Conservación Internacional Centroamérica, Mohamed Bin Zayed Conservation Fund, Marine Action Conservation Fund (MACF) y Columbus Zoo, sin cuyos fondos no hubiera sido posible realizar esta investigación. Por último agradecer al Dr. Maurizicio Protti de la Universidad Nacional de Costa Rica, quien enriqueció este trabajo con su experiencia, comentarios y sugerencias. Se agradece el Proyecto Consolidación de Áreas Marinas Protegidas del Sistema Nacional de Áreas de Conservación, Programa de Naciones Unidas para el Desarrollo y el Global Environment Facility (GEF) por su colaboración en la publicación.

\section{RESUMEN}

De mayo 2010 a mayo 2011 se recolectó información referente a 67 faenas pesqueras artesanal con línea de fondo (44.8\% observaciones abordo y $55.2 \%$ observaciones de descargas en centros de acopio) realizadas en Golfo Dulce, Pacífico Sur de Costa Rica. Durante las 67 faenas de pesca se analizaron en total 609 tiburones pertenecientes a diez especies. De estas, sobresalen S. lewini (más de 51.8\% del total de tiburones analizados), M. lunulatus (23.5\%), $R$. longurio, (13.8\%) y C. limbatus (8.9\%). Otras especies G. cirratum, G. cuvier, N.velox, S. tiburo y H. mexicanus fueron muy esporádicas ya que aportaron cerca del $2.0 \%$ del total. La condición de captura, el bajo valor comercial y la disponibilidad de los pescadores, permitió liberar con vida y sin anzuelos un total de 65 tiburones y 111 rayas, de las cuales $75.7 \%$ fueron rayas látigo (D. longa), $12.6 \%$ rayas gavilana (A. narinari), 5.4\% rayas nariz de vaca $(R$. steindachneri), 5.4\% rayas guitarra ( $R$. leucorhynchus), $2.7 \%$ rayas de puntos ( $U$. chilenis), $0.9 \%$ rayas mariposa (G. marmorata) y $0.9 \%$ mobula (Mobula sp.). Los análisis de CPUE en Golfo Dulce revelaron las mayores CPUE de tiburones en julio-agosto, mientras las mayores capturas de rayas se presentaron en enero-febrero.

Palabras clave: Golfo Dulce, pesquería artesanal, tiburones, rayas, observaciones pesqueras, descargas.

\section{REFERENCIAS}

Amador, L. (2010). Descripción y análisis de la pesca artesanal de la raya látigo Dasyatis longa (Dasyatidae) en Tárcoles, Puntarenas, Pacífico de Costa Rica (Tesis de licenciatura). Escuela de Biología, Universidad Nacional de Costa Rica, Heredia, Costa Rica.

Anislado, T. V. (2000). Ecología pesquera del tiburón martillo Sphyrna lewini (Griffith Y Smith, 1834), en el litoral del estado de Michoacán, México (Tesis maestría). Universidad Nacional Autónoma de México, México.

Arauz, R., López A., \& Zanella I. (2007). Análisis de la descarga anual de tiburones y rayas en la pesquería pelágica y costera del Pacífico de Costa Rica (Playas del Coco, Tárcoles y Golfito) (Informe final). San José, Costa Rica: Unpublished.

Arauz, R., López, A., Zanella, I., Bolaños, A., \& Suarez, R. (2008). Análisis de las capturas y descargas de 
tiburones en las pesquerías del Pacifico de Costa Rica (Playas del Coco y Golfito). San José, Costa Rica: Conservación Internacional Centroamérica.

Arauz, R., López, A., Zanella, I., \& Bolaños, A. (2009). Acción por los tiburones: Análisis de las capturas y avistamientos de elasmobranquios en las aguas costeras y pelágicas del Pacífico de Costa Rica (Informe final). San José, Costa Rica: Unpublished

Branstetter, S. (1987). Age, growth and reproductive biology of the silky shark, Carcharhinus falciformis, and the scalloped hammerhead, Sphyrna lewini, from thenorthwestern Gulf of Mexico. Environmental Biology of Fishes, 19(3), 161-173.

Campana, S. E., Marks, L., Joyce, W., \& Kohler, N. E. (2006). Effects of recreational and commercial fishing on blue sharks (Prionace glauca) in Atlantic Canada, with inferences on the North Atlantic population. Canadian Journal of Fisheries and Aquatic Sciences, 63, 670-682.

Campos, J. (1989). Evaluación de la pesca artesanal del Golfo Dulce (Proyecto cooperativo Ministerio de Agricultura y Ganadería - Universidad de Costa Rica). San José, Costa Rica: CIMAR, Universidad de Costa Rica.

Camhi, M. (1999). Sharks on the Line: Analysis of Pacific State Shark Fisheries. New York, USA: Living Oceans Program, National Audubon Society, Islip.

Carlson, J. K., Goldman, K. J., \& Lowe, C. C. (2004). Metabolism, Energetic demand and Endothermy. In J. C. Carrier, J. A. Musick, \& M. R. Heithaus (Eds.), Biology of sharks and their relatives (pp. 203-224). Boca Raton, Florida, USA: CRC Press.

Clarke, S. C., Harley, S. J., Hoyle, S. D., \& Rice, J. S. (2013). Population Trends in Pacific Oceanic Sharks and the Utility of Regulations on Shark Finning. Conservation Biology, 27(1), 197-209.

Dean, H. (1996). Pollychaete Works (Annelida) collected in Golfo Dulce, during theVictor Hensen Costa Rica expedition (1993/1994). Revista de Biología Tropical, 44(3), 81-86.

Di Rienzo, J. A., Casanoves, F., Balzarini, M. G., Gonzalez, L., Tablada, M., \& Robledo, C. W. (2009). InfoStat (Versión 2009). Universidad Nacional de Córdoba, Argentina: Grupo InfoStat, FCA.

Fargier, L., Hartmann, H., \& Molina, H. (2008). Desarrollo de un proceso de monitoreo participativo en las comunidades pesqueras de Bahía Pavones (Golfo Dulce) en el Pacifico Sur de Costa Rica (Informe). San José, Costa Rica: Unpublished.

Fischer, W., Krupp, F., Schneider, W., Sommer, C., Carpenter, K. E., \& Niem, V. H. (1995). Guía FAO para la Identificación de Especies para los Fines de la Pesca, Pacífico centro-oriental, Plantas e Invertebrados
(Vol. I). Roma, Italia: Organización de las Naciones Unidas para la Agricultura y la Alimentación (FAO).

Gaitán, J., \& López, A. (2008). Presence of young blacktip sharks Carcharhinus limbatus (Carcharhiniformes: Carcharhinidae) in the north area of the Tayrona ecoregion, Colombian Caribbean. Latin American Journal of Aquatic Research, 36(1), 115-119.

Guzmán, A., \& Molina, H. (2007). La Pesquería Artesanal de Peces en Golfo Dulce, Costa Rica. Punta Cana, Republica Domenicana, 60th Gulf and Caribbean Fisheries Institute.

Hueter R., Castillo, L., Márquez, F., \& Tyminski, J. (2007). The Use of Laguna Yalahau, Quintana Roo, Mexico as a Primary Nursery for the Blacktip Shark. American Fisheries Society Symposium, 50, 345-364.

Kohler, N. E., Casey, J. G., \& Turne, P. A. (1996). LengthLength and Length-Weight Relationships for 13 Shark Species from the Western North Atlantic (NOAA Technical Memorandum NMFS-NE-110). Massachusetts, USA, Northeast Fisheries Science Center, Woods Hole.

La Gaceta. (2010). Decreto INCOPESCA AJDPI/251. Diario Oficial, Costa Rica. $N^{\circ} 159$, Versión Digital. San José, Costa Rica.

López, J. (2009). Aspectos Biológicos de la raya látigo Dasyatis longa (Pisces: Dasyatidae) de la zona central del Pacífico colombiano (Trabajo de grado). Facultad de Ciencias, Universidad del Valle Facultad de Ciencias, Santiago de Cali, Colombia.

López, A. (2012). Identificación, evaluación y manejo de hábitats críticos utilizados por el tiburón martillo, Sphyrna lewini, y otros elasmobranquios en Golfo Dulce, Costa Rica (Tesis de maestría). Universidad Nacional, Heredia, Costa Rica.

López, A., Arauz R., Zanella, I., \& Le Foulge, L. (2009). Análisis anual de las capturas de tiburones y rayas en las pesquerías artesanales de Tárcoles, Pacífico Central de Costa Rica. Revista de Ciencias Marinas y Costeras, 1, 145-157.

López, A., \& Zanella, I. (2011). Conservación del tiburón martillo (Sphyrna lewini en Golfo Dulce (Informe para Conservación Internacional). San José, Costa Rica: Unpublished.

Márquez, J. F., Corro, D., \& Castillo, J. F. (2005). Observations on the Biology of Pacific Sharpnose Shark (Rizhoprionodon longurio, Jordan and Gilbert, 1882), Captured in Souther Sinaloa, México. Journal of Northwest Atlantic Fishery Science, 35, 107-114.

Martínez, D., Montero, A., \& May-Collado, L. (2011). Cetáceos de las aguas costeras del Pacífico Norte y Sur de Costa Rica. Revista de Biología Tropical, 59(1), 283-290.

Maunder, M. N., Sibert, J. R., Fonteneau, A., Hampton, J., Kleiber, P., \& Harley, S. J. (2006). Interpreting catch 
per unit effort data to assess the status of individual stocks and communities. ICES Journal of Marine Science, 63, 1373-1385.

Mohanraj, G., Rajapakiam, S., Mohan, S., Batcha, H., \& Gomathy, S. (2009). Status of Elasmobranchs Fishery in Chennai, India. Asian Fisheries Science, 22, 607-615.

Morales, A. (2011). La diversidad marina del Golfo Dulce, Pacífico Sur de Costa Rica: amenazas a su conservación. Biocenosis, 24(1-2), 9-20.

Morgan, A., \& Carlson, J. K. (2010). Capture time, size, and hooking mortality of bottom longline-caught sharks. Fisheries Research, 101, 32-37.

Musick, J., \& Bonfil, R. (2004). Elasmobranch fisheries management techniques. Singapore, Japan: APEC Fisheries Working Group.

Navia, A., Giraldo, A., \& Mejía, P. (2006). Nota Científica: Notas sobre la biología y dieta del toyo vieja (Mustelus lunulatus) en la zona central de pesca del Pacífico colombiano. Investigaciones Marinas, 34(2), 217-222.

Pérez-Jiménez, J. C., Sosa-Nishizak, O., \& Estrada, E. F. (2005). Artisanal Shark Fishery at "Tres Marias" Islands and Isabel Island in the Central Mexican Pacific. Journal of Northwest Atlantic Fishery Science, 35, 333-343.

Quesada, M., \& Alfaro, E. (2004). Comportamiento de las masas de agua en el Golfo Dulce, Costa Rica, durante el Niño (1997-1998) Revista de Biología Tropical, 52(2), 95-103.
Quirós, G. (2003). Circulación del Golfo Dulce: un fiordo tropical. Tópicos Meteorológicos y Oceanográficos, 10(2), 75-83.

Robinson, L., \& Sauer, W. H. H. (2013). A first description of the artisanal shark fishery in northern Madagascar: implications for management. African Journal of Marine Science, 35(1), 9-15.

Skomal, G. B. (2007). Evaluating the physiological and physical consequences of capture on post-release survivorship in large pelagic fishes. Fisheries Management \& Ecology, 14, 81-89.

Sierra, C., Vartanian, D., \& Polimeni, J. (2003). Caracterización social, económica y ambiental del Área de Conservación Osa. San José, Costa Rica: Dirección de la Sociedad Civil, MINAE.

Smith, W. D., Bizzarro, J. J., \& Cailliet, G. M. (2009). La pesca artesanal de elasmobranquios en la costa oriental de Baja California, México. Ciencias Marinas, 35(2), 209-236.

Villavicencio, C. J. (1995). Distribución temporal y condición reproductiva de las rayas (Pisces: Batoidei), capturadas comercialmente en Bahía Almejas, B.C.S., México. Revista de Investigación Científica-Área de Ciencias del mar, 6(1-2), 1-12.

Villavicencio, C., Downton, C., \& Meléndez, E. (1994). Tamaño y reproducción de la raya Dasyatis longus (Pisces: Dasyatidae), en Bahía Almejas, Baja California Sur, México. Revista de Biología Tropical, $42,375-377$. 